\title{
The Evolution of Achalasia
}

\author{
Donald O. Castell
}

Received: 28 November 2011 / Accepted: 1 December 2011/Published online: 20 December 2011

(C) Springer Science+Business Media, LLC 2011

Esophageal motility abnormalities have been described as existing in a spectrum which includes achalasia, distal esophageal spasm, nutcracker esophagus and possibly other less distinct motility findings. There has been much questioning and controversy relevant to whether these different manometric features evolve from one to another and thus form a changing spectrum amongst themselves. Most clear in the reported literature extending back into the 1960s are reports of esophageal spasm "evolving" into more typical achalasia over time [1]. What has never been established, however, is whether a less clear form of motility defect can be identified that will predictably evolve into classic achalasia. It is intuitive, however, that the patient with a normal esophagus when exposed to the etiologic agent for achalasia (currently unknown) would show a progression (evolution?) of manometric changes over time.

The term non-specific esophageal motility disorder (NEMD) was introduced into the literature in an attempt to establish a category for abnormal motility that did not quite fit the classic definitions for achalasia, spasm and nutcracker esophagus. Since it was described to include all motility abnormalities that fail to fit more classic definitions, the potential for a broad and truly non-specific group of findings was established. Thus, NEMD became a wastebasket for potential motility changes not otherwise well described. In the mid 1990s, when we surveyed a group of some 60 patients diagnosed with NEMD, we recognized that essentially $100 \%$ of them actually had been placed in this category based on the findings of increased

D. O. Castell ( ()

Department of Gastroenterology and Hepatology, Medical University of South Carolina, Charleston, SC 29425-2900, USA

e-mail: castell@musc.edu numbers of low amplitude "ineffective" peristaltic waves as described in 1988 by the manofluoroscopic studies of Kahrilas et al. [2]. We suggested that the term "ineffective esophageal motility" (IEM) was a more meaningful descriptor for this ill-defined manometric entity and that it would better define the motility findings previously called "non-specific" [3].

In this issue of Digestive Diseases and Sciences, Müller et al. [4] suggest that patients previously diagnosed with NEMD had a high likelihood of evolving to classic achalasia. This may well be true, however, there are aspects of their report that one must consider before accepting this as a fact. Included in these are questions whether the initial manometric diagnosis was correct or did a number of these patients initially have findings that would actually be consistent with a diagnosis of achalasia? This is particularly pertinent when one recognizes the fact that the so called "classic" manometric findings of achalasia, a hypertensive resting LES with poor relaxation and the absence of peristalsis, may only all be found in less than one-third of otherwise typical cases [5].

The suggestion that approximately $50 \%$ of NEMD patients might evolve into typical achalasia seems exceedingly high, but would certainly be expected if the initial interpretation was incorrect and the diagnosis was actually a form of achalasia. Furthermore, if the 15 achalasia cases described by Müller et al. are based on the original 76 NEMD patients, then the incidence of achalasia falls to $19 \%$. This raises the second important question involving these data. Since only 28 of the original 76 patients had a repeat manometry, it is quite likely that a serious selection bias is in play here. My guess would be that many of these actually were patients with early achalasia when their initial studies were performed and they were self-selected based on the continuing progression 
of their symptoms. It is hardly fair to suggest a $50 \%$ likelihood of progression to definitive achalasia with follow-up of this kind. In our experience with patients with typical IEM followed for over 12 months with repeat manometry we found that $90 \%$ of them continued to show the IEM pattern and none progressed to achalasia (Castell et al., personal communication). Low amplitude non-peristaltic contractions are, in fact, the hallmark of classic achalasia. Such findings can well be confused with a possible diagnosis of NEMD. We regularly include a full barium esophagram in our diagnostic evaluation of patients with equivocal manometric findings as supporting information, particularly if achalasia is suspected.

Do I believe that a high percentage of patients with "non-specific" findings on their esophageal motility testing will develop into achalasia? I think not, since it is not consistent with my experience of greater than 40 years as an "esophagologist". It would be a mistake to take a leap of faith to this particular conclusion without further investigation and more routine follow-up in other series of these patients. However, we should thank Müller and colleagues for having the tenacity to prospectively follow these patients and to bring to our attention a possible manometric evolution.

\section{References}

1. Kramer P, Harris L, Donaldson R. Transition from symptomatic diffuse spasm to cardiospasm. Gastroenterology. 1967;8:115.

2. Kahrilas PJ, Dodds WJ, Hogan WJ. Effect of peristaltic dysfunction on esophageal volume clearance. Gastroenterology. 1988;94: 73-80.

3. Leite LP, Johnston BT, Barrett J, Castell JA, Castell DO. Ineffective esophageal motility (IEM): the primary finding in patients with non-specific esophageal motility disorder. Dig Dis Sci. 1997;42:1853-1858.

4. Müller M, Eckardt AJ, Göpel B, Eckardt VF. Clinical and manometric course of nonspecific esophageal motility disorders. Dig Dis Sci. (Epub ahead of print). doi:10.1007/s10620-0111937-y.

5. Agrawal A, Hila A, Tutuian R, Castell DO. Manometry and impedance characteristics of achalasia. Facts and myths. J Clin Gastroenterol. 2008;42:266-270. 